\title{
Vadim Kuznetsov. Informal Biography by Eyes of His First Adviser ${ }^{\star}$
}

Igor V. KOMAROV

\author{
St. Petersburg State University, St. Petersburg, Russia \\ E-mail: komarov@pobox.spbu.ru
}

Received May 20, 2007; Published online June 22, 2007

Original article is available at http://www.emis.de/journals/SIGMA/2007/074/

\begin{abstract}
The paper is dedicated to the memory of prominent theoretical physicist and mathematician Dr. Vadim Kuznetsov who worked, in particular, in the fields of the nonlinear dynamics, separation of variables, integrability theory, special functions. It includes his short research biography, an account of the start of his research career and the list of publications.
\end{abstract}

Key words: classical and quantum integrable systems; separation of variables

2000 Mathematics Subject Classification: 01A70

Vadim Kuznetsov was a classical "self-made man", nobody of his family ever earned their living by intellectual work. Vadim's talent and will was the main source of his achievements and authority among the professionals.

The external canvas of his biography looks streamlined: the Leningrad University, the postgraduate course at the same university, defence of the thesis and work of a researcher. In April 1993 Vadim got his first post-doctoral position (Department of Mathematics, University of Amsterdam, a NWO post-doctoral research fellow involved in the project "Special functions and quantum inverse scattering method" supervised by Prof. T.H. Koornwinder). In 1996 V. Kuznetsov spent six months at the Technical University in Lyngby, Denmark, where he lectured in classical and quantum integrable systems. That was followed by two months of work at the Centre de recherches mathématiques, Université de Montréal as a research fellow. In 1996 Vadim Kuznetsov finally settled in Leeds (UK) were he worked at the university, first on the grant basis and then at a permanent position.

In 1980 Vadim Kuznetsov entered the Department of Physics of the Leningrad University, where since 1982 he had chosen the major at the department of quantum mechanics. I was a lecturer then at the same department, and I had to give a course with a strange title "Introduction to Profession". The students had not studied quantum mechanics at that moment, and it was not clear what should be taught. The administration told me to do what I wished to. I have chosen a system of talks based on book then recently published in Russian by W. Miller "Separation of Variables". Some of the students were given papers in other topics. Vadim got a paper from Journal of the Optical Society of America on halo effect at atmospheric light scattering on ice crystals. With respect to the talk, Vadim showed persistence in studying of mathematical and physical details of the paper. Then I got an impression of him as an accurate person who is good in laborious tasks. Later I asked Vadim whether this course had any influence upon him. The answer was negative. Maybe because of this course Vadim did not feel any "trepidation" with respect to the classical theory of separation of variables.

In around a year Vadim overtook me in a stairway and asked for a topic for research. On my way I suggested him to find a semi-classical spectrum of the Kowalevski top. I had two ideas - to use Kolosov's projection of the Kowalevski problem onto a flat potential problem and

${ }^{\star}$ This paper is a contribution to the Vadim Kuznetsov Memorial Issue 'Integrable Systems and Related Topics'. The full collection is available at http://www.emis.de/journals/SIGMA/kuznetsov.html 
to apply the adiabatic switching method for finding of the spectrum. I believed then that it was a sure-win problem needing only labor input. We started regular and intense discussions on this topic. In a few months Vadim brought me integrals of action calculated in line with the Kolosov's method. I asked him to investigate limiting cases for weak and strong fields. In one of the limits one degree of freedom was disappearing. The formulas were cumbersome, and I believed that to be a result of some technical mistake. We got stuck at that place for several months. Gradually it became clear that the reason for the problem is that the Kolosov's transformation where time also transforms is not canonical. Vadim Kuznetsov found an indication how to proceed correctly in the paper by S.P. Novikov and A.P. Veselov: first of all, Kowalevski variables should have been transformed into the Poisson commuting ones. We invented the next step ourselves. It was restoration of the canonical variables when the integrals of motion in the Lagrangian variables were known. For me and probably for Vadim that was a very happy moment. A student in his very first work started to generate professional ideas at the serious level and got assured in his research capacities.

Approximately at the same time E.K. Sklyanin included the Goryachev-Chaplygin gyrostat I have studied into the framework of the quantum inverse scattering method (QISM). The Sklyanin's method had various names - "the functional Bethe ansatz", "the magical recipe". It was obvious that it dealt with finding of the separation of variables (SoV) in the QISM. I brought V.B. Kuznetsov and his classmate A.V. Tsiganov to L.D. Fadeev's seminar at the Leningrad Branch of Steklov Mathematical Institute and introduced them to E.K. Sklyanin. V. Kuznetsov thoroughly studied each new paper by E.K. Sklyanin. In particular, some time later Vadim studied the algebra of the reflection equations (we called it then QISM II), and he was the first to construct the separation for one example of QISM II.

At that time Vadim needed to complete technically his education and to defend a master thesis on the basis of the results already obtained. It became clear to us that development and application of the separation of variables in the QISM is a priority task. It was much later when in the paper [43] "Kowalevski top revisited" in 2002 Vadim got back to the top problem and found explicit and quite cumbersome expressions for $2 \times 2$ of the Lax matrix for the Kowalevski top. The results of his master thesis were published in [1] in Theoretical and Mathematical Physics in 1987.

In 1986-1989 Vadim was a post-graduate student at the department of computational physics to which I moved some time before. Our meetings were regular and fruitful, Vadim was quickly becoming a qualified and independent researcher. I set some problems for him, and Vadim found some problems himself. We also discussed strategic issues - in what direction it is preferable to continue research in future. Vadim responded positively to extension of the list of the integrable systems, incorporation of the known integrable systems into the QISM schemes, accumulation of the variable separation experience in particular cases. On the opposite, Vadim rejected my suggestion to look inside the QISM for efficient algorithms for finding the spectrum of the integrals of motion that were naturally related to the QISM. Among the "consumers" of the new ideas of the SoV method, the one mentioned most often was the theory of special functions that we perceived in the spirit of the three-volume book by Bateman and Erdely.

Several Lax-like representations for the Kowalevski problem appeared in the 1980-ies. Vadim rewrote the available direct derivation of the separated equations for the case $s o(4, \mathbb{C})$ in the spirit of Heine-Horozov. At the same time A.G. Reiman and M.A. Semenov-Tyan-Shanskii constructed $4 \times 4$ and $5 \times 5$ Lax matrices for the original Kowalevski problem. The new Lax matrices depreciated the previous variants and allowed simplification of the classical equations of motion in terms of Prym $\theta$-functions. The attempts to find separation of variables from the new Lax matrices did not lead to any success. As Vadim pointed out later [43] (Kowalevski top revisited, 2002), until then there was only one separation of variables known for this problem following from original Kowalevski's papers. Vadim had identified in a $4 \times 4$ minor of the 
Lax matrix for the Kowalevski problem a new $3 \times 3$ Lax matrix for the Goryachev-Chaplygin gyrostat. With A.I. Bobenko they had applied this matrix for integration of the respective equations of motion [3].

After the post-graduate course and defence of his thesis (referees E.K. Sklyanin and A.R. Its) earlier than the scheduled date in October of 1989 V. Kuznetsov worked as a researcher at the Department of Computational Physics.

In autumn of 1991 Ernie Kalnins that worked for many years on the coordinate separation of variables visited our university. Ernie wanted to see Riga, and Vadim accompanied him on that trip. Before Ernie Kalnins's visit Vadim wrote on his own initiative several papers on co-ordinate separation of variables for free motion in the spaces of permanent curvature in classical and quantum mechanics on the basis of currents algebra [9, 10, 11]. The most important was that it was possible not only to reproduce quite transparently the known results on co-ordinate separation of variables for the Laplace operators in the low-dimensional spaces of constant curvature from the Gaudin algebra, but also to extend this procedure to the spaces with arbitrary number of dimensions and to construct reduction of quadrics. These results attracted the considerable interest at the conference in Obninsk of N.Ya. Vilenkin who asked questions to Vadim for quite a long time and in great detail. Later (February 25, 1993) Vadim received for this cycle of papers the Award of the Academiae Europaeae (London), instituted by the club of the Russian members of the Academiae for young Russian scientists.

The end of the 1980-ies and the beginning of the 1990-ies were a difficult time for survival. The USSR economy was collapsing fast. The researcher's salary was evidently insufficient to support the family. Vadim found odd jobs, up to petty trade near metro stations that was very humiliating for him. Vadim found a postdoctoral position at the University of Amsterdam. I learned about his decision to go abroad when Vadim asked me for a letter of recommendation. In Amsterdam Vadim worked together with the expert on the special functions theory Thom H. Koornwinder. The results of their joint research were included into the 1994 papers [18, 25] were properties of the Gauss hypergeometric function (in particular, contiguous function relations) were derived in a regular way from the properties of the $R$-matrix algebra. The papers of this period developed the ideas of the separation of variables for rational and trigonometric linear and quadratic $R$-matrix algebra.

The list of Vadim's co-authors was extending fast. Vadim advanced his knowledge and mastered new fields of mathematics and theoretical physics.

In 1994 a many-year co-operation of V.B. Kuznetsov and E.K. Sklyanin had started. They obtained separation of variables for the $A_{2}$ Jack polynomials. Systematic research in the field of the Ruijsenaars-Schneider model was also started [26] (the co-authors were F.W. Nijhoff, O. Ragnisco, E.K. Sklyanin). I would like to mention here the observation on the relation of the Bethe equations with the integrable time-discretisation of the model.

It is known that for the separation of variables by means of the poles of the Baker-Akhiezer function $\mathbf{f}$ its special normalisation is needed. For many examples the scalar product $(\mathbf{f}, \mathbf{a})$ with a constant vector a was sufficient, but sometimes the number of poles exceeded the number of degrees of freedom. In the paper on $\mathcal{D}_{n}$ type periodic Toda lattice V. Kuznetsov managed to find a correct normalising vector a depending on the dynamic variables [30]. But that was his last work involving poles of the Baker-Akhiezer function. Presence of artificial tricks made Vadim to look for more universal approaches.

The papers by V.B. Kuznetsov and E.K. Sklyanin [32] developed a new more systematic approach to separation of variables and presented relation of the SoV with the Bäklund transformation. Invention of the "spectrality" property was a key point. The Baxter $Q$-operator became a subject of attention of many researchers. Vadim often said that a new understanding of the separation of variables started for him from the 1998 paper [32] "On Bäcklund transformations 
for many-body systems". Development of this technique by the group of mathematicians with participation of V. Kuznetsov led to qualitatively new results on factorisation of the symmetric Jack, Hall-Littlewood and Macdonald polynomials.

For more than 10 years Vadim maintained research contacts with the group of Mark Adler, Pierre van Moerbeke and Pol Vanhaecke, with whom he published only two papers [40,41] on geometric aspects of the Bäcklund transform.

In 2000 Vadim organised jointly with Frank Nijfoff the International Workshop on Mathematical Methods of Regular Dynamics dedicated to the 150th anniversary of Sonya Kowalevski. In 2002 Vadim Kuznetsov and Frank Nijhoff edited the materials of the Workshop [42]. In 2003 Vadim was an organiser of the workshop in Edinburgh that was dedicated to the classical works by H. Jack, P. Hall, D.E. Littlewood and I.G. MacDonald on Symmetric Functions and their relation to Representation Theory of Symmetric Groups. The materials of this workshop edited by V. Kuznetsov and S. Sahi [51] were published after his death. In that issue Brian D. Sleeman and Evgeny K. Sklyanin published a paper dedicated to Vadim Kuznetsov's memory ${ }^{1}$.

Vadim worked a lot directly with formulas. Sometimes he obtained results like "Hidden symmetry of the quantum Calogero-Moser system", 1996, that at the first glance had no relation to the general theory. This list can be extended and it may give start to future studies.

I visited Vadim and his family in Amsterdam, Copenhagen and Leeds, and we also met during Vadim's short visits to St. Petersburgh and Dubna. In July 1998 both of us were in different regions of Germany and specially came to Kaizerslautern to see each other. We were walking in the city where some Sunday German Fest was bustling, and Vadim enthusiastically spoke about new ideas in the joint publications with E.K. Sklyanin. Later the research aspect of our discussions moved to a background. During our last meetings in Leeds in May 2005 Vadim looked very tired, he has immense teaching and administrative load. We tried to talk about mathematics without big success. Vadim mentioned that he intended to start a completely new research area not related to separation of variables. I know nothing about implementation of these plans.

Vadim was survived by his wife Olga and son Simon.

\section{Dr. Vadim B. Kuznetsov. List of publications}

[1] Komarov I.V., Kuznetsov V.B., Quasiclassical quantization of the Kowalevski top, Teoret. Mat. Fiz. 73 (1987), 335-347 (English transl.: Theor. and Math. Phys. 73 (1987), 1255-1263).

[2] Komarov I.V., Kuznetsov V.B., The generalized Goryachev-Chaplygin gyrostat in quantum mechanics, in Differentsialnaya Geom. Gruppy Li i Mekh., Vol. IX, Zap. Nauchn. Sem. Leningrad. Otdel. Mat. Inst. Steklov. (LOMI) 164 (1987), 133-141, 198 (English transl.: J. Soviet Math. 47 (1989), no. 2, 2459-2466).

[3] Bobenko A.I., Kuznetsov V.B., Lax representation and new formulae for the Goryachev-Chaplygin top, J. Phys. A: Math. Gen. 21 (1988), 1999-2006.

[4] Kuznetsov V.B., Tsiganov A.V., A special case of Neumann's system and the Kowalevski-ChaplyginGoryachev top, J. Phys. A: Math. Gen. 22 (1989), L73-L79.

[5] Kuznetsov V.B., Tsiganov A.V., Infinite series of Lie algebras and boundary conditions for integrable systems, in Differentsialnaya Geom. Gruppy Li i Mekh., Vol. 10, Zapiski Nauchn. Sem. LOMI 172 (1992), 88-98, 170 (English transl.: J. Soviet Math. 59 (1992), no. 5, 1085-1092).

[6] Komarov I.V., Kuznetsov V.B., Kowalevski's top on the Lie algebras o(4), e(3), and o(3,1), J. Phys. A: Math. Gen. 23 (1990), 841-846.

[7] Kuznetsov V.B., Generalized polyspheroidal periodic functions and the quantum inverse scattering method, J. Math. Phys. 31 (1990), 1167-1174.

[8] Komarov I.V., Kuznetsov V.B., Quantum Euler-Manakov top on the 3-sphere $S_{3}$, J. Phys. A: Math. Gen. 24 (1991), L737-L742.

\footnotetext{
${ }^{1}$ Sleeman B.D., Sklyanin E.K., Vadim Borisovich Kuznetsov 1963-2005, Contemp. Math. 417 (2006), 357-360.
} 
[9] Kuznetsov V.B., On the equivalence of two families of quantum integrable systems, in Voprosy Kvant. Teor. Polya Statist. Fiz., Vol. 11, Zap. Nauchn. Sem. S.-Peterburg. Otdel. Mat. Inst. Steklov. (POMI) Vol. 199 (1992), 114-131, 186 (English transl.: J. Math. Sci. 77 (1995), no. 2, 3090-3101).

[10] Kuznetsov V.B., Quadrics on Riemannian spaces of constant curvature. Separation of variables and a connection with the Gaudin magnet, Teoret. Mat. Fiz. 91 (1992), no. 1, 83-111 (English transl.: Theoret. and Math. Phys. 91 (1992), no. 1, 385-404).

[11] Kuznetsov V.B., Quadrics on real Riemannian spaces of constant curvature: separation of variables and connection with Gaudin magnet, J. Math. Phys. 33 (1992), 3240-3254.

[12] Kuznetsov V.B., On isomorphism of $n$-dimensional Neumann system and $n$-site Gaudin magnet, Funktsional. Anal. i Prilozhen. 26 (1992), no. 4, 88-90 ((English transl.: Funct. Anal. Appl. 26 (1992), no. 4, 302-304).

[13] Kuznetsov V.B., Equivalence of two graphical calculi, J. Phys. A: Math. Gen. 25 (1992), 6005-6026.

[14] Enol'skii V.Z., Kuznetsov V.B., Salerno M., On the quantum inverse scattering method for the DST dimer, Phys. D 68 (1993), 138-152.

[15] Eilbeck J.C., Enol'skii V.Z., Kuznetsov V.B., Leykin D.V., Linear $r$-matrix algebra for systems separable in parabolic coordinates, Phys. Lett. A 180 (1993), 208-214.

[16] Christiansen P.L., Jørgensen M.F., Kuznetsov V.B., On integrable systems close to the Toda lattice, Lett. Math. Phys. 29 (1993), 165-173.

[17] Kuznetsov V.B.; Tsyganov A.V., Quantum relativistic Toda lattices, in Differentsialnaya Geom. Gruppy Li i Mekh., Vol. 13, Zap. Nauchn. Sem. S.-Peterburg. Otdel. Mat. Inst. Steklov. (POMI) 205 (1993), 71-84, 180 (English transl.: J. Math. Sci. 80 (1996), no. 3, 1802-1810).

[18] Koornwinder T.H., Kuznetsov V.B., Gauss hypergeometric function and quadratic R-matrix algebras, Algebra i Analiz 6 (1994), 161-184 (English transl.: St. Petersburg Math. J. 6 (1995), 595-618), hep-th/9311152.

[19] Kalnins E.G., Kuznetsov V.B., Miller W.Jr., Quadrics on complex Riemannian spaces of constant curvature, separation of variables and the Gaudin magnet, J. Math. Phys. 35 (1994), 1710-1731, hep-th/9308109.

[20] Eilbeck J.C., Enol'skii V.Z., Kuznetsov V.B., Tsiganov A.V., Linear $r$-matrix algebra for classical separable systems, J. Phys. A: Math. Gen. 27 (1994), 567-578, hep-th/9306155.

[21] Eilbeck J.C., Enol'skii V.Z., Kuznetsov V.B., Leykin D.V., Classical Poisson structure for a hierarchy of one-dimensional particle systems separable in parabolic coordinates, J. Nonlinear Math. Phys. 1 (1994), 275-294.

[22] Kalnins E.G., Kuznetsov V.B., Miller W.Jr., Separation of variables and XXZ Gaudin magnet, Rend. Sem. Mat. Univ. Politec. Torino 53 (1995), 109-120, hep-th/9412190.

[23] Kuznetsov V.B., Jørgensen M.F., Christiansen P.L., New boundary conditions for integrable lattices, J. Phys. A: Math. Gen. 28 (1995), 4639-4654, hep-th/9503168.

[24] Kuznetsov V.B., Sklyanin E.K., Separation of variables in $A_{2}$ type Jack polynomials, RIMS Kokyuroku 919 (1995), 27-43, solv-int/9508002.

[25] Kuznetsov V.B., ${ }_{3} F_{2}(1)$ hypergeometric function and quadratic $R$-matrix algebra, in Symmetries and Integrability of difference equations (Esterel, PQ, 1994), CRM Proc. Lecture Notes, Vol. 9, Amer. Math. Soc., Providence, RI, 1996, 185-197, hep-th/9410146.

[26] Nijhoff F.W., Ragnisco O., Kuznetsov V.B., Integrable time-discretization of the Ruijsenaars-Schneider model, Comm. Math. Phys. 176 (1996), 681-700, hep-th/9412170.

[27] Kuznetsov V.B., Hidden symmetry of the quantum Calogero-Moser system, Phys. Lett. A 218 (1996), 212-222, solv-int/9509001.

[28] Kuznetsov V.B., Sklyanin E.K., Separation of variables for the $A_{2}$ Ruijsenaars system and a new integral representation for the $A_{2}$ Macdonald polynomials, J. Phys. A: Math. Gen. 29 (1996), 2779-2804, q-alg/9602023.

[29] Nijhoff F.W., Kuznetsov V.B., Sklyanin E.K., Ragnisco O., Dynamical $r$-matrix for the elliptic RuijsenaarsSchneider system, J. Phys. A: Math. Gen. 29 (1996), L333-L340, solv-int/9603006.

[30] Kuznetsov V.B., Separation of variables for the $\mathcal{D}_{n}$ type periodic Toda lattice, J. Phys. A: Math. Gen. 30 (1997), 2127-2138, solv-int/9701009.

[31] Kuznetsov V.B., Nijhoff F.W., Sklyanin E.K., Separation of variables for the Ruijsenaars system, Comm. Math. Phys. 189 (1997), 855-877, solv-int/9701004.

[32] Kuznetsov V.B., Sklyanin E.K., On Bäcklund transformations for many-body systems, J. Phys. A: Math. Gen. 31 (1998), 2241-2251, solv-int/9711010. 
[33] Kuznetsov V.B., Sklyanin E.K., Separation of variables and integral relations for special functions, Ramanujan J. 3 1999, no. 1, 5-35, q-alg/9705006.

[34] Kuznetsov V.B., Sklyanin E.K., Factorisation of Macdonald polynomials, in Symmetries and Integrability of Difference Equations (Canterbury, 1996), London Math. Soc. Lecture Note Ser., Vol. 255, Cambridge Univ. Press, Cambridge, 1999, 370-384, q-alg/9703013.

[35] Hone A.N.W., Kuznetsov V.B., Ragnisco O., Bäcklund transformations for many-body systems related to KdV, J. Phys. A: Math. Gen. 32 (1999), L299-L306, solv-int/9904003.

[36] Hone A.N.W., Kuznetsov V.B., Ragnisco O., Bäcklund transformations for the Henon-Heiles and Garnier systems, in SIDE III - Symmetries and Integrability of Difference Equations (Sabaudia, 1998), CRM Proc. Lecture Notes, Vol. 25, Amer. Math. Soc., Providence, RI, 2000, 231-235.

[37] Kuznetsov V.B., Salerno M., Sklyanin E.K., Quantum Bäcklund transformation for the integrable DST model, J. Phys. A: Math. Gen. 33 (2000), no. 1, 171-189, solv-int/9908002.

[38] Kuznetsov V.B., Nijhoff F.W. (Editors), Kowalevski workshop on mathematical methods of regular dynamics, Papers from the workshop held at the University of Leeds (Leeds, April 12-15, 2000), J. Phys. A: Math. Gen. 34 (2001), no. 11.

[39] Hone A.N.W., Kuznetsov V.B., Ragnisco O., Bäcklund transformations for the sl(2) Gaudin magnet, J. Phys. A: Math. Gen. 34 (2001), 2477-2490, nlin.SI/0007041.

[40] Kuznetsov V.B., Vanhaecke P., Bäcklund transformations for finite-dimensional integrable systems: a geometric approach, J. Geom. Phys. 44 (2002), 1-40, nlin.SI/0004003.

[41] Adler M., Kuznetsov V.B., van Moerbeke P., Rational solutions to the Pfaff lattice and Jack polynomials, Ergodic Theory Dynam. Systems 22 (2002), 1365-1405, nlin.SI/0202037.

[42] Kuznetsov V.B. (Editors), The Kowalevski property, Proceedings of the Kowalevski Workshop on Mathematical Methods of Regular Dynamics (MMRD), dedicated to the 150th anniversary of Sophie Kowalevski's birth (University of Leeds, April 12-15, 2000), CRM Proceedings and Lecture Notes, Vol. 32, Amer. Math. Soc., Providence, RI, 2002.

[43] Kuznetsov V.B., Kowalevski top revisited, in The Kowalevski Property (Leeds, 2000), CRM Proc. Lecture Notes, Vol. 32, Amer. Math. Soc., Providence, RI, 2002, 181-196, nlin.SI/0110012.

[44] Kuznetsov V.B., Simultaneous separation for the Kowalevski and Goryachev-Chaplygin gyrostats, J. Phys. A: Math. Gen. 35 (2002), 6419-6430, nlin.SI/0201004.

[45] Kuznetsov V.B., Inverse problem for SL(2) lattices, in SPT 2002: Symmetry and Perturbation Theory (Cala Gonone), World Sci. Publishing, River Edge, NJ, 2002, 136-152, nlin.SI/0207025.

[46] Kuznetsov V.B., Mangazeev V.V., Sklyanin E.K., Q-operator and factorised separation chain for Jack polynomials, Indag. Math. (N.S.) 4 (2003), 451-482, math.CA/0306242.

[47] Kuznetsov V.B., Petrera M.,, Ragnisco O., Separation of variables and Bäcklund transformations for the symmetric Lagrange top, J. Phys. A: Math. Gen. 37 (2004), 8495-8512, nlin.SI/0403028.

[48] Yuzbashyan E.A., Altshuler B.L., Kuznetsov V.B., Enolskii V.Z., Solution for the dynamics of the BCS and central spin problems, J. Phys. A: Math. Gen. 38 (2005), 7831-7849, cond-mat/0407501.

[49] Yuzbashyan E.A., Altshuler B.L., Kuznetsov V.B., Enolskii V.Z., Nonequilibrium Cooper pairing in the nonadiabatic regime, Phys. Rev. B 72 (2005), 220503(R), 4 pages, cond-mat/0505493.

[50] Yuzbashyan E.A., Kuznetsov V.B., Altshuler B.L., Integrable dynamics of coupled Fermi-Bose condensates, Phys. Rev. B $\mathbf{7 2}$ (2005), 144524, 9 pages, cond-mat/0506782.

[51] Kuznetsov V.B., Sahi S. (Editors), Jack, Hall-Littlewood and Macdonald polynomials, Proceedings of the Workshop held in Edinburgh (September 23-26, 2003), Contemp. Math. 417 (2006).

[52] Kuznetsov V.B., Sahi S., Preface, in Jack, Hall-Littlewood and Macdonald Polynomials, Editors V.B. Kuznetsov and S. Sahi, Contemp. Math. 417 (2006), ix-xvii.

[53] Kuznetsov V.B., Sklyanin E.K., Factorization of symmetric polynomials, in Jack, Hall-Littlewood and Macdonald Polynomials, Editors V.B. Kuznetsov and S. Sahi, Contemp. Math. 417 (2006), 239-256, math.CA/0501257.

[54] Kuznetsov V.B., Sklyanin E.K., Eigenproblem for Jacobi matrices: hypergeometric series solution, math.CO/0509298.

[55] Kuznetsov V.B., Orthogonal polynomials and separation of variables, in Orthogonal Polynomials and Special Functions, Lecture Notes in Math., Vol. 1883, Springer, Berlin, 2006, 229-254.

[56] Kuznetsov V.B., Sklyanin E.K., Bäcklund transformation for the BC-Type Toda lattice, SIGMA 3 (2007), 080, 17 pages, arXiv:0707.1950. 